\title{
Base de données sur la qualité des rejets urbains de temps de pluie (QASTOR) : distribution de la pollution rejetée, dimensions des ouvrages d'interception
}

\author{
par Agnès Saget*, Ghassan Chebbo \\ CERGRENE, La Courtine, 93167 Noisy-le-Grand Cedex
}

\section{I 圆 INTRODUCTION}

Les rejets urbains par temps de pluie, à savoir les déversoirs de réseaux unitaires et les rejets à l'exutoire des réseaux séparatifs, entraînent des dégradations importantes dans les milieux récepteurs où ils sont rejetés sans traitement préalable. Jusqu'aux années 1970 , les rejets étaient considérés uniquement du point de vue hydraulique. Il fallait évacuer les eaux le plus vite possible loin des zones habitées. Leur aspect pollué a longtemps été négligé, mais il a bien fallu constater que les milieux récepteurs en souffraient. Aussi, des études ont-elle commencé à être mises en place pour étudier les rejets qui engendraient des impacts néfastes aux milieux récepteurs. Des campagnes de mesures ont alors été menées un peu partout dans le monde. Elles avaient pour but de mieux connaître les caractéristiques des rejets. Mais sans cadre général dans lequel s'inscrire, elles voulaient répondre à des questions locales et immédiates, avec des méthodes d'exploitation hétérogènes qui se sont avérées par la suite parfois très approximatives. C'est dans ce cadre que s'inscrit l'élaboration de la base de données QASTOR (Qualité en Assainissement par Temps d'ORage) : rassembler les données existantes et les exploiter de manière globale. Le projet a été mené au CERGRENE avec la participation financière de l'Agence de l'Eau Seine-Normandie.

Dans cet article nous présentons successivement :

- la base de données Qastor et son contenu;

- les résultats obtenus concernant la pollution rejetée pendant un an, pendant les événements les plus importants et la répartition au cours des événements ;

- les résultats obtenus concernant la dimension des ouvrages de traitement.

\section{PRÉSENTATION DE QASTOR}

L'intérêt de QASTOR est de regrouper des données françaises sur un même support et de les exploiter avec des méthodes identiques et de manière globale, afin d'obtenir des informations sur la variabilité des résultats. De plus, le coût élevé pour acquérir des données rendent celles qui exis- tent très précieuses et justifient l'effort qui a été fait pour les conserver et les valoriser. Enfin, la récupération des données ne s'est pas faite sans mal et a montré un manque d'unité dans l'expression des mesures. Certaines d'entre elles ont été acquises il y a plus de 20 ans, un certain nombre d'informations ont donc été très difficilement récupérables, ou sous des formes très diverses. Il a fallu fournir un important travail pour rendre les données homogènes et les mettre au même format. Une base de données constitue alors une sorte de référence à laquelle faire appel pour rendre homogène l'acquisition de données dans ce domaine.

Les données nous ont été fournies par la Direction de l'eau et de l'assainissement de la Seine Saint Denis, le Laboratoire régional de l'ouest parisien et le Service technique de l'urbanisme. Aujourd'hui, QASTOR rassemble $17 \mathrm{cam}$ pagnes de mesures par temps de pluie de longue durée (entre 10 et 16 mois), sur des réseaux unitaires et séparatifs, pour lesquelles on dispose simultanément d'informations sur la pluie, sur les écoulements en réseau à l'exutoire des bassins versants et sur la qualité des rejets aux mêmes points. Il existe néanmoins des lacunes sur les volumes écoulés et les concentrations moyennes par événement qui doivent être reconstituées avant d'effectuer les calculs.

La vérification et la critique des données, essentielles avant toute utilisation, a permis d'attribuer à chaque mesure (pluie précipitée, débit écoulé et concentration de polluant) une note de validité, qui permet d'estimer si la mesure est douteuse ou correcte.

\section{DISTRIBUTION DE LA POLLUTION REJETÉE}

\subsection{Présentation}

L'ensemble des informations récoltées a été exploité de manière globale. Le premier objectif de la base de données

\footnotetext{
* Agnès Saget a obtenu le prix Henri Milon.
} 
Tableau 1. - Caractéristiques des campagnes de mesures contenues dans QASTOR.

\begin{tabular}{|c|c|c|c|c|c|}
\hline \multirow[b]{2}{*}{ Site } & \multirow{2}{*}{$\begin{array}{c}\text { Surface } \\
\text { totale } \\
\text { ha }\end{array}$} & \multirow[b]{2}{*}{$\begin{array}{c}\text { Surface active* } \\
\text { ha }\end{array}$} & \multicolumn{3}{|c|}{ Nombre de mesures } \\
\hline & & & Pluie & $\begin{array}{l}\text { Volume total } \\
\text { (Hydrogram) }\end{array}$ & $\begin{array}{c}\text { Concentration } \\
\text { moyenne } \\
\text { (Pollutogram) }\end{array}$ \\
\hline $\begin{array}{l}\text { Séparatif } \\
\text { Aix Zup } \\
\text { Aix Nord } \\
\text { Maurepas } \\
\text { Vélizy } \\
\text { Centre Urbain } \\
\text { Ulis Sud } \\
\text { Malnoue }\end{array}$ & $\begin{array}{c}256 \\
92 \\
267 \\
532 \\
226 \\
691 \\
185\end{array}$ & $\begin{array}{l}12 \\
12 \\
15 \\
20 \\
24 \\
27 \\
29\end{array}$ & $\begin{array}{c}73 \\
72 \\
156 \\
141 \\
130 \\
130 \\
110\end{array}$ & $\begin{array}{c}73(73) \\
66(66) \\
172(171) \\
23(19) \\
72(5) \\
74(10) \\
100(9)\end{array}$ & $\begin{array}{l}54(20) \\
52(12) \\
130(24) \\
27(19) \\
25(5) \\
31(10) \\
26(9)\end{array}$ \\
\hline $\begin{array}{l}\text { Sites séparatifs } \\
\text { pollués et mixtes } \\
\text { Ulis Nord } \\
\text { Grammont } \\
\text { Chesnay } \\
\text { Baudile }\end{array}$ & $\begin{array}{c}431 \\
144 \\
560 \\
1145\end{array}$ & $\begin{array}{c}16 \\
25 \\
46 \\
197\end{array}$ & $\begin{array}{c}92 \\
120 \\
155 \\
151\end{array}$ & $\begin{array}{c}93(91) \\
96(7) \\
50(5) \\
78(5)\end{array}$ & $\begin{array}{c}81(14) \\
27(7) \\
22(5) \\
27(5)\end{array}$ \\
\hline $\begin{array}{l}\text { Sites unitaires } \\
\text { Mantes } \\
\text { la Briche DD11 } \\
\text { la Briche D11 } \\
\text { la Briche PHI } \\
\text { la Briche Enghien } \\
\text { la Briche PLB }\end{array}$ & 72 & $\begin{array}{c}7,6 \\
467 \\
657 \\
694 \\
1380 \\
4600\end{array}$ & $\begin{array}{l}234 \\
89 \\
89 \\
89 \\
89 \\
89\end{array}$ & $\begin{array}{l}25(9) \\
16(16) \\
17(17) \\
18(18) \\
17(17) \\
18(18)\end{array}$ & $\begin{array}{l}25(10) \\
16(16) \\
17(17) \\
18(18) \\
17(17) \\
18(18)\end{array}$ \\
\hline
\end{tabular}

* Volume mesuré pendant la campagne / Hauteur de pluie correspondante.

est de fournir des éléments pour une caractérisation plus poussée de la pollution rejetée, à différentes échelles de temps, de quelques minutes ou quelques heures, à plusieurs mois. Ces évaluations sont importantes pour étudier les impacts sur les milieux récepteurs afin de fournir des données d'entrée qui pourront éclaircir quels sont les rejets qui créent les impacts. La base permet de rechercher des indicateurs qui expliqueront les variabilités et par là même la formation de la pollution. Sur le long terme, on a évalué les masses annuellement rejetées. Sur le court terme, on a tenté d'identifier les caractéristiques des événements pluvieux les mieux reliées aux concentrations et aux masses de pollution rejetées par événement. On a également porté un intérêt tout particulier aux événements les plus pollués. A une échelle de temps encore plus fine, on a étudié la répartition de la masse de pollution rejetée en cours d'écoulement.

\subsection{Les masses annuelles}

Pour chaque bassin versant, les données disponibles couvrent approximativement un an. Mais il n'y a pas de mesure de pollution pour chaque événement de la série. Pour estimer la masse de pollution rejetée pendant un an, on a besoin de la masse rejetée pendant chaque événement de l'année. - Par conséquent les données manquantes ont dû être reconstituées. Pour cela, des équations de régression ont été établies à partir des données disponibles. Elles représentent la meilleure relation linéaire entre les masses de pollution et certaines caractéristiques des événements pluvieux. Ces caractéristiques sont choisies parmi les suivantes : hauteur d'eau précipitée, intensité moyenne sur $5 \mathrm{~min}$, sur le temps de concentration, durée de la pluie, durée de temps sec antécédent, hauteur précipitée pendant l'événement précédent, volume total écoulé, débit moyen et débit de pointe.

On a établi une équation par bassin versant. Ces équations sont très différentes d'un site à un autre, on ne peut pas trou- ver une variable commune à l'ensemble des équations. La méthode de calcul de la régression permet d'estimer un intervalle de confiance autour d'une valeur calculée pour un événement. Par suite, on peut estimer un intervalle de confiance autour de la masse annuelle. Pour certaines des valeurs annuelles, ces intervalles sont très larges. D'autre part, les coefficients de corrélation, qui témoignent de la qualité de l'ajustement, varient dans de grandes proportions et sont d'ailleurs parfois assez faibles. Dans ces cas, les valeurs manquantes ont simplement été remplacées par une valeur moyenne au lieu d'utiliser une équation de régression plus fiable.

D'après les valeurs du tableau 2 , on constate que les variations entre les sites sont beaucoup plus importantes que les variations entre les types de réseau. On suppose qu'en fait les variations sont dues aux différences existant entre les années de mesure. En effet, les mesures n'ont pas été faites pendant la même année sur les différents bassins, et les périodes de retour des événements ne sont pas comparables. Pour mieux connaître l'influence des caractéristiques des sites, on a besoin des mesures sur plusieurs années avec des régimes pluviométriques différents.

Toutefoiș, afin d'expliquer les variations observées, les masses annuelles sont exprimées par rapport à des caractéristiques du site comme le nombre des habitants. On a également regardé les relations entre les masses annuelles et les pentes moyennes ou les temps de concentration du bassin. Mais aucune relation n'apparaît. Les intervalles de confiance sont très larges et peuvent masquer les possibles relations.

\subsection{Les masses rejetées au cours des événements les plus polluants}

Afin d'avoir une idée de la masse de pollution qui peut être déversée au cours d'un événement unique, de chaque série de mesures on a extrait les valeurs de masses et de concen- 
Tableau 2. - Ordre de grandeur des masses : par an, par an et par hectare actif, par an et par habitant, par mm d'eau précipitée et concentration moyenne.

\begin{tabular}{|c|c|c|c|c|c|c|c|}
\hline \multirow{3}{*}{\multicolumn{2}{|c|}{ Pollution rejetée }} & \multicolumn{6}{|c|}{ Type de réseau } \\
\hline & & \multicolumn{2}{|c|}{ Séparatif } & \multicolumn{2}{|c|}{ Séparatif pollué } & \multicolumn{2}{|c|}{ Unitaire } \\
\hline & & $\begin{array}{l}\text { valeur } \\
\text { estimée }\end{array}$ & $\begin{array}{c}\text { intervalle de } \\
\text { confiance } 90 \%\end{array}$ & $\begin{array}{l}\text { valeur } \\
\text { estimée }\end{array}$ & $\begin{array}{l}\text { intervalle de } \\
\text { confiance } 90 \%\end{array}$ & $\begin{array}{l}\text { valeur } \\
\text { estimée }\end{array}$ & $\begin{array}{l}\text { intervalle de } \\
\text { confiance } 90 \%\end{array}$ \\
\hline MES & $\begin{array}{l}\text { tonne } \\
\mathrm{kg} / \mathrm{ha} \text { act } \\
\mathrm{kg} / \mathrm{hab} \\
\mathrm{kg} / \mathrm{mm} \\
\mathrm{mg} / \mathrm{l}\end{array}$ & $\begin{array}{c}17-180 \\
1300-6700 \\
4,8-8,6 \\
18-227 \\
160-460 \\
\end{array}$ & $\begin{array}{c}17-200 \\
1000-7400 \\
4-8,6\end{array}$ & $\begin{array}{c}41-660 \\
1800-4800 \\
2,7-13,1 \\
63-580 \\
240-400 \\
\end{array}$ & $\begin{array}{c}41-680 \\
1700-6700 \\
2,4-13,5\end{array}$ & $\begin{array}{c}25-10800 \\
1700-4300 \\
5,2 \text { (Mantes) } \\
64-17600 \\
240-670\end{array}$ & $\begin{array}{c}25-14 \quad 100 \\
1200-4 \quad 400\end{array}$ \\
\hline DCO & $\begin{array}{l}\text { tonne } \\
\mathrm{kg} / \mathrm{ha} a c t \\
\mathrm{~kg} / \mathrm{hab} \\
\mathrm{kg} / \mathrm{mm} \\
\mathrm{mg} / \mathrm{l}\end{array}$ & $\begin{array}{c}10-130 \\
670-4500 \\
3,2-12,1 \\
8,3-68 \\
80-320\end{array}$ & $\begin{array}{c}10-150 \\
670-5200 \\
2,8-14\end{array}$ & $\begin{array}{c}20-360 \\
1250-5200 \\
1,3-7,1 \\
30-610 \\
180-470\end{array}$ & $\begin{array}{c}20-410 \\
1200-7400 \\
1,3-8,1\end{array}$ & $\begin{array}{c}14-13200 \\
1550-4200 \\
2,9 \text { (Mantes) } \\
36-21000 \\
350-570\end{array}$ & $\begin{array}{c}14-15200 \\
1050-4200\end{array}$ \\
\hline DBO5 & $\begin{array}{l}\text { tonne } \\
\mathrm{kg} / \mathrm{ha} \text { act } \\
\mathrm{kg} / \mathrm{hab} \\
\mathrm{ka} / \mathrm{mm} \\
\mathrm{mg} / \mathrm{l}\end{array}$ & $\begin{array}{c}1,5-13 \\
100-450 \\
0,58-1,20 \\
1,2-29 \\
13-130\end{array}$ & $\begin{array}{c}1,5-15 \\
100-520 \\
0,56-1,40\end{array}$ & $\begin{array}{c}3,8-120 \\
240-1000 \\
0,25-2,38 \\
5,7-270 \\
35-120\end{array}$ & $\begin{array}{c}3,8-120 \\
240-1400 \\
0,25-2,4\end{array}$ & $\begin{array}{c}6,2-5080 \\
810-1490 \\
1,3 \text { (Mante) } \\
8,8-9400 \\
90-270\end{array}$ & $\begin{array}{l}5,9-6500 \\
780-1530\end{array}$ \\
\hline
\end{tabular}

trations les plus importantes. Les résultats sont consignés dans le tableau 3, mais ils ne représentent qu'un exemple de ce qui peut être rejeté pendant un événement pluvieux. En effet, ces valeurs ne sont pas les plus fortes valeurs possibles, ce sont les plus fortes valeurs parmi les séries mesurées. Par conséquent, les différences observées reflètent probablement les différences entre les événements, leurs caractéristiques sont très différentes que ce soit en terme de hauteur de pluie ou d'intensité. De plus, il est impossible de leur donner une période de retour. Néanmoins, les valeurs du tableau 3 soulignent que la pollution rejetée pendant quelques heures peut atteindre presque $30 \%$ de la masse rejetée pendant l'année. Pour les milieux récepteurs, ce type d'événements entraînent des effets de choc très destructeurs. Cela met en évidence l'effort particulier qu'il faut pour protéger les eaux réceptrices et pour définir les objectifs à atteindre dans la protection contre des événements particuliers ou contre l'accumulation des polluants pendant plusieurs mois.
3.4 Répartition des charges polluantes à l'intérieur d'un événement pluvieux : notion du premier flot d'orage

La notion de premier flot suppose que la première fraction du volume écoulé contient la plus grande part de la pollution transportée au cours d'un événement pluvieux. Pour le moment il n'existe pas de définition rigoureuse de cette notion. Les enjeux sont pourtant importants puisqu'il s'agit de déterminer la taille des ouvrages de traitement des rejets urbains par temps de pluie, et plus particulièrement celle des bassins de stockage-décantation. En effet, si le premier flot, c'est-à-dire la première fraction du volume écoulé, contient la plus grande part de la charge polluante transportée au cours d'un événement pluvieux, il suffira d'intercepter le volume correspondant au premier flot pour intercepter la majeure partie de la pollution et protéger efficacement le milieu naturel.

Tableau 3. - Les événements les plus polluants (ordre de grandeur des masses totales, divisées par la surface et pourcentage des masses annuelles et ordre de grandeur des concentrations).

\begin{tabular}{|c|c|c|c|c|}
\hline \multirow{2}{*}{ Polluant } & \multirow{2}{*}{$\begin{array}{l}\text { Rejet le plus } \\
\text { important }\end{array}$} & \multicolumn{3}{|c|}{ Type de réseau } \\
\hline & & Séparatif & Séparatif pollué & Unitaire \\
\hline MES & $\begin{array}{l}\mathrm{kg} \\
\mathrm{kg} / \mathrm{ha} \text { act } \\
\% \mathrm{M} \text { an } \\
\mathrm{mg} / \mathrm{l}\end{array}$ & $\begin{array}{c}1500-10500 \\
80-550 \\
6-27 \\
645-3800\end{array}$ & $\begin{array}{c}2300-16200 \\
80-180 \\
2-8 \\
780-2500\end{array}$ & $\begin{array}{c}1500-144000 \\
24-190 \\
1-17 \\
740-1800\end{array}$ \\
\hline $\mathrm{DCO}$ & $\begin{array}{l}\mathrm{kg} \\
\mathrm{kg} / \mathrm{ha} \text { act } \\
\% \mathrm{M} \text { an } \\
\mathrm{mg} / \mathrm{l}\end{array}$ & $\begin{array}{c}840-3800 \\
56-310 \\
5-28 \\
500-1500\end{array}$ & $\begin{array}{c}100-17400 \\
15-215 \\
5-10 \\
900-2700\end{array}$ & $\begin{array}{c}580-165000 \\
32-75 \\
2-5 \\
840-1 \quad 100\end{array}$ \\
\hline DBO5 & $\begin{array}{l}\mathrm{kg} \\
\mathrm{kg} / \mathrm{ha} \text { act } \\
\% \mathrm{M} \text { an } \\
\mathrm{mg} / \mathrm{l}\end{array}$ & $\begin{array}{c}175-580 \\
8-90 \\
5-15 \\
50-750\end{array}$ & $\begin{array}{c}160-7700 \\
10-39 \\
5-10 \\
250-820\end{array}$ & $\begin{array}{c}55-62300 \\
7-31 \\
2-5 \\
250-480\end{array}$ \\
\hline
\end{tabular}


Pour permettre une étude comparative des évolutions des flux polluants pour différents événements pluvieux, on utilise une représentation adimensionnelle qui consiste à tracer la courbe donnant l'évolution de la charge polluante cumulée divisée par la charge polluante totale en fonction du volume cumulé divisé par le volume total; on note ce type de courbe $M(V)$. Toute courbe $M(V)$ peut être représentée par une fonction puissance du type $: F(X)=X^{b}$ définie sur l'intervalle $[0,1]$, et telle que $F(0)=0$ et $F(1)=1$. Il est très facile de déterminer la valeur du paramètre $b$ par une corrélation linéaire puisque: $F(X)=X^{b} \Leftrightarrow \log (F(X))=b \log (X)$.

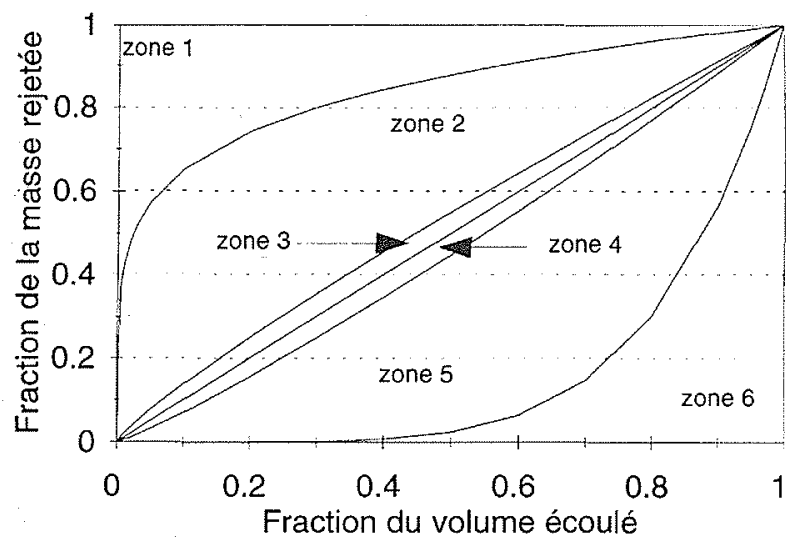

1. Zones des courbes $M(V)$ en fonction du paramètre $b$.

La valeur du paramètre $b$ caractérise l'écart entre la courbe $M(V)$ et la bissectrice, qui représente le cas où la concentration est constante en cours d'écoulement $(b=1)$. Le premier flot est défini par les trois propositions équivalentes suivantes:

- la valeur de $b$ est inférieure à 0,185 ,

- la courbe $M(V)$ passe dans la zone 1 lorsque $X \leq 0,30$,

- plus de $80 \%$ de la charge polluante sont transportés dans les premiers $30 \%$ du volume.

Le premier flot est quantifié par la valeur du paramètre $b$ : lorsque $b$ est inférieur à 0.185 , plus $b$ est faible et plus le premier flot est important.
Des ajustements ont été effectués pour obtenir pour chaque événement la valeur du coefficient $b$. Il apparaît à la lecture du tableau 5 que, sur les événements disponibles, le phénomène de premier flot, tel que nous l'avons défini c'està-dire lorsque les courbes sont dans la zone 1 , est quasiment inexistant. La situation extrême opposée, à savoir la zone 6 , est également inexistante.

Pour les sites séparatifs, la majorité des événements se situent dans la zone 2, ce qui traduit un écart positif moyen au-dessus de la bissectrice. En revanche, pour les sites unitaires, les événements paraissent également répartiș entre les zones 2,3 et 4 . Dans ces deux dernières zones, on peut considérer que les courbes sont peu différentes de la bissectrice.

Une quantification précise du phénomène de premier flot, puis son application à des données réelles, montre que c'est un phénomène rare, trop rare pour pouvoir établir à partir de son existence une stratégie efficace de lutte contre la pollution des rejets urbains par temps de pluie.

\section{IV 噃 DIMENSIONS DES OUVRAGES DE TRAITEMENT}

\subsection{Présentation}

La prise de conscience des problèmes engendrés par les rejets urbains a conduit à une prise en compte également au niveau législatif. Il y a eu en France la nouvelle loi sur l'eau en 1992, et il y a des directives européennes sur la qualité des eaux. Pour répondre aux normes de qualité, il faut mettre en place des ouvrages de traitement. Or à l'heure actuelle, on sait peu de choses sur les dimensions qu'ils doivent avoir pour capter une fraction suffisante de la pollution. Le deuxième objectif de l'exploitation de la base est de fournir des éléments sur les dimensions que doivent avoir les ouvrages de traitement. Jusqu'à présent dans la littérature, lorsque des études sur des ouvrages de traitement sont présentées, il s'agit du fonctionnement interne des ouvrages et de leur rendement d'épuration en comparant les masses de pollution qui entrent à celles qui sortent. On a peu étudié l'efficacité d'interception des ouvrages, en comparant la pollution qui entre dans l'ouvrage avec celle qui est produite

Tableau 4. - Caractéristiques des courbes $M(V)$ en fonction du paramètre $b$.

\begin{tabular}{|c|c|c|c|c|}
\hline \multicolumn{2}{|c|}{ Valeur du paramètre $b$} & \multirow{2}{*}{$\begin{array}{c}\text { Zone } \\
1 \\
2 \\
3\end{array}$} & \multicolumn{2}{|c|}{ Ecart de $M(V)$ par rapport à la bissectrice } \\
\hline$b<1$ & $\begin{array}{l}0<b \leq 0,185 \\
0,185<b \leq 0,862 \\
0,862<b \leq 1,000\end{array}$ & & écart positif & $\begin{array}{l}\text { fort } \\
\text { moyen } \\
\text { négligeable }\end{array}$ \\
\hline$b>1$ & $\begin{array}{l}1,000<b \leq 1,159 \\
1,159<b \leq 5,395 \\
5,395<b<+\infty\end{array}$ & $\begin{array}{l}4 \\
5 \\
6\end{array}$ & écart négatif & $\begin{array}{l}\text { négligeable } \\
\text { moyen } \\
\text { fort }\end{array}$ \\
\hline
\end{tabular}

Tableau 5. - Pourcentages d'événements appartenant aux zones définies dans la figure 1.

\begin{tabular}{|c|c|c|c|c|c|c|c|c|}
\hline \multirow{2}{*}{ Paramètre } & \multirow{2}{*}{ Type de site } & \multicolumn{6}{|c|}{$\%$ d'événements dans les zones } & \multirow{2}{*}{$\begin{array}{l}\text { Nombre total } \\
\text { d'événements }\end{array}$} \\
\hline & & 1 & 2 & 3 & 4 & 5 & 6 & \\
\hline MES & $\begin{array}{l}\text { séparatif } \\
\text { unitaire }\end{array}$ & $\begin{array}{l}1 \\
0\end{array}$ & $\begin{array}{l}66 \\
33\end{array}$ & $\begin{array}{l}14 \\
32\end{array}$ & $\begin{array}{l}11 \\
25\end{array}$ & $\begin{array}{l}8 \\
9\end{array}$ & $\begin{array}{l}0 \\
0\end{array}$ & $\begin{array}{l}80 \\
117\end{array}$ \\
\hline $\mathrm{DCO}$ & $\begin{array}{l}\text { séparatif } \\
\text { unitaire }\end{array}$ & $\begin{array}{l}0 \\
0\end{array}$ & $\begin{array}{l}82 \\
50\end{array}$ & $\begin{array}{l}11 \\
33\end{array}$ & $\begin{array}{c}3 \\
14\end{array}$ & $\begin{array}{l}4 \\
3\end{array}$ & $\begin{array}{l}0 \\
0\end{array}$ & $\begin{array}{c}80 \\
117\end{array}$ \\
\hline DBO5 & $\begin{array}{l}\text { séparatif } \\
\text { unitaire }\end{array}$ & $\begin{array}{l}0 \\
0\end{array}$ & $\begin{array}{l}82 \\
57\end{array}$ & $\begin{array}{c}9 \\
25\end{array}$ & $\begin{array}{c}5 \\
15\end{array}$ & $\begin{array}{l}4 \\
2\end{array}$ & $\begin{array}{l}0 \\
0\end{array}$ & $\begin{array}{l}77 \\
91\end{array}$ \\
\hline
\end{tabular}


sur l'ensemble du bassin versant, sans entrer dans le détail du fonctionnement des ouvrages.

Qualitativement on sait que plus les masses déversées sont importantes, plus les impacts sont importants. Nous n'avons pas d'indications sur les milieux récepteurs dans lesquels ont lieu les rejets étudiés, et nous ne pouvons pas chiffrer les seuils au-dessus desquels les masses de pollution rejetées deviennent néfastes. Par conséquent, nous ne pouvons pas raisonner sur la pollution que rejette un ouvrage, mais uniquement sur les fractions interceptées exprimées en pourcentage de la pollution produite pendant les événements pluvieux. D'autre part, les études d'impact sur les milieux ont mis en évidence l'influence des échelles de temps pendant lesquelles interviennent les rejets. Les effets négatifs peuvent se classer en deux catégories : à long terme (des effets cumulatifs concernent les polluants difficilement dégradables et qui peuvent être très toxiques, comme par exemple les métaux lourds) et à court terme (des effets de choc sont provoqués par des arrivées brutales et massives de pollution qui entrainent par exemple des consommations brutales d'oxygène dissous). Nous avons défini deux efficacités d'interception pour évaluer l'influence des ouvrages sur ces effets :

- pendant une série d'événements pour les effets à long terme : rapport de la somme des masses de pollution interceptées et la somme des masses totales produites.

- pendant un événement pour les effets à court terme : rapport entre la masse de pollution interceptée pendant l'événement et la masse totale de l'événement.

On suppose que les ouvrages de stockage sont vides au début de chaque pluie, et que leur remplissage intervient depuis le début de l'écoulement jusqu'à ce qu'ils soient pleins. Pendant un événement, si le volume de l'ouvrage est supérieur au volume écoulé pendant l'événement, alors la masse interceptée représente la totalité de la masse produite. Sinon, la masse interceptée se lit sur la courbe qui donne la répartition de la masse de pollution cumulée depuis le début de l'écoulement en fonction du volume écoulé au cours de l'événement. Lorsque cette courbe manque, on utilise des courbes extraites de l'ensemble des courbes mesurées. Ce sont les courbes à 25 et $75 \%$, c'est-à-dire qu'entre la courbe inférieure et la courbe 25 ou 75 se situent respectivement 25 ou $75 \%$ des courbes mesurées. On considère que la vraie courbe se situe entre les deux.

Pour l'estimation des débits de traitement, les calculs n'ont pu être effectués que sur quatre sites pour lesquels les données disponibles sont adéquates. En effet, pour ce cas il faut les données des hydrogrammes pour mener les calculs.

\subsection{Interception sur une série d'événements}

Les résultats sont exprimés par hectare actif, car diviser les valeurs par la surface active permet de comparer les différents sites. Pour les deux types d'ouvrages, ils montrent une forte dispersion. Ainsi, pour intercepter $80 \%$ de la masse rejetée pendant les séries, il faut des volumes compris entre 30 et $150 \mathrm{~m}^{3} / \mathrm{ha}$ actif et des débits entre 11 et $60 \mathrm{l} / \mathrm{s} / \mathrm{ha}$ actif.
Lorsqu'on distingue les différents types de réseau, les volumes nécessaires sur les sites unitaires semblent plus faibles que sur les sites séparatifs. En effet, pour intercepter $80 \%$ de la masse, les valeurs sont les suivantes:

- pour les sites séparatifs, entre 60 et $150 \mathrm{~m}^{3} / \mathrm{ha}$ actif,

- pour les sites unitaires, entre 30 et $70 \mathrm{~m}^{3} / \mathrm{ha}$ actif.

Dans le paragraphe 3 , on a montré que la fréquence du phénomène de premier flot est très rare. En tout cas, la pollution des premiers flots sur les sites unitaires n'est pas plus importante que sur les sites séparatifs. Par conséquent, ce n'est pas la répartition de la pollution à l'intérieur des événements qui explique les différences entre sites unitaires et séparatifs, observées sur les volumes à intercepter. Cela provient plus probablement des différences sur les événements utilisés. En effet, les campagnes de mesures ont été faites à des périodes différentes (de 1974 à 1983). Sur ces années, la pluviométrie annuelle correspondant aux campagnes utilisées varie entre 600 et $900 \mathrm{~mm}$.

En ce qui concerne le volume total, les domaines de variation se recoupent en partie sur les différents types de réseau. Néanmoins, les volumes les plus faibles correspondent aux sites unitaires alors que les volumes les plus forts correspondent aux sites séparatifs. La différence sur les volumes à intercepter peut donc provenir en partie de la différence sur les volumes écoulés.

Pour les volumes maximaux, les domaines de variation entre sites unitaires et séparatifs ne se recouvrent pas du tout, car les volumes correspondent à des événements qui n'ont pas la même période de retour. Leur influence sur les volumes à intercepter semble très importante. Les volumes d'interception semblent plus faibles sur les sites unitaires car sur ces sites les volumes mesurés sont également plus faibles. Autrement dit les volumes d'interception semblent liés au type d'événement mesuré pendant la campagne.

Pour le moment sur les sites utilisés, nous ne savons pas à quoi correspond une pluviométrie moyenne, dont la connaissance pourrait être obtenue à partir d'une très longue série de pluies. Comme les volumes des ouvrages dépendent fortement des caractéristiques des événements utilisés, il est impossible d'indiquer quels volumes doivent être retenus pour intercepter la masse de pollution produite lors d'une année de pluviométrie moyenne.

\subsection{Pour les événements les plus pollués}

Afin d'identifier les événements qu'il faut traiter en priorité, nous avons extrait de chaque série ceux pour lesquels les masses produites sont les plus grandes. Ces valeurs ramenées à la surface active sont très variables; elles sont comprises entre 20 et $400 \mathrm{~kg} / \mathrm{ha}$ actif. En fait, elles représentent des événements différents et de période de retour différente (tabl.6 et 7). Les masses peuvent être très grandes, et elles ne sont pas négligeables par rapport à la masse annuelle (plus de $15 \%$ de la masse annuelle dans $30 \%$ des cas). Et s'il est possible de traiter ces événements de manière efficace, la protection du milieu sera assurée contre les effets de choc.

Tableau 6. - Volume écoulé pendant l'ensemble des événements utilisés, volume écoulé le plus grand pendant un événement, et volume pour intercepter $80 \%$ de la masse de pollution produite à long terme.

\begin{tabular}{|l|c|c|c|}
\hline Site & $\begin{array}{c}\text { Volume total } \mathbf{~ m}^{\mathbf{3}} \mathbf{h a} \\
\text { actif }\end{array}$ & $\begin{array}{c}\text { Volume maximal } \\
\mathbf{m}^{\mathbf{3}} \mathbf{/ h a} \text { actif }\end{array}$ & $\begin{array}{c}\text { Volume à intercepter } \\
\mathbf{m}^{\mathbf{3} / \mathbf{h a}} \mathbf{a c t i f}\end{array}$ \\
\hline Séparatif & $4800-11100$ & $480-840$ & $63-153$ \\
Séparatif (Aix-en-Provence) & $5420-5750$ & $558-562$ & $64-83$ \\
Séparatif pollué & $5040-9900$ & $410-700$ & $58-150$ \\
Unitaire & $3800-8300$ & $90-220$ & $31-68$ \\
\hline
\end{tabular}


Tableau 7. - Débit le plus grand pendant un événement, et débit pour intercepter $80 \%$ de la masse de pollution produite à long terme.

\begin{tabular}{|l|c|c|}
\hline Site & Débit de pointe I/s/ha actif & Débit à traiter $\mathbf{~ / s / h a ~ a c t i f ~}$ \\
\hline Aix Zup & 200 & $11-12$ \\
Aix Nord & 360 & $58-59$ \\
Maurepas & 190 & $16-17$ \\
Ulis Nord & 72 & $11-12$ \\
\hline
\end{tabular}

Concernant les volumes de stockage, lorsque l'événement est caractérisé simultanément par les volume écoulé et masse de pollution les plus forts de la série observée, l'interception de $80 \%$ de la masse correspond à un volume supérieur à celui nécessaire pour intercepter $80 \%$ de la masse de la série correspondante (de 60 à $800 \mathrm{~m}^{3} /$ ha actif contre 30 à $150 \mathrm{~m}^{3} / \mathrm{ha}$ actif). Si, en revanche, la masse la plus grande de la série ne correspond pas au volume maximal de cette série, alors la capacité à réserver est plus modeste (entre 20 et $250 \mathrm{~m}^{3} /$ ha actif).

Concernant les débits de traitement, les efficacités d'interception pour les événements dont les masses sont les plus fortes sont très variables (entre 3 et $200 \mathrm{l} / \mathrm{s} / \mathrm{ha}$ actif). Lorsque la masse produite la plus forte correspond au débit de pointe le plus grand, le débit nécessaire pour traiter la pollution est important. Lorsque les deux maximaux ne sont pas observés en même temps, c'est l'événement pour lequel le débit de pointe est maximum qui rejette le plus.

Les événements les plus polluants ne correspondent pas aux mêmes périodes de retour. Il est donc difficile de comparer les résultats. D'autre part, intercepter l'événement pour lequel la masse de pollution est la plus grande n'est pas nécessairement un objectif pertinent. En effet, c'est la masse rejetée qui détermine la capacité de l'ouvrage, et donc le mode de fonctionnement de cet ouvrage au regard de la distribution simultanée de la pluie et de la pollution.

\section{$\mathrm{V}$ 置 CONCLUSIONS}

L'exploitation de la base de données QASTOR a permis de foumir des informations intéressantes sur la production de la pollution par temps de pluie. Les résultats obtenus sont toutefois étonnants dans la mesure où ils ne permettent pas de confirmer certaines idées concernant la production de la pollution, comme par exemple l'importance de la durée de temps sec précédant un événement.

Tout d'abord, les résultats obtenus présentent une grande variabilité. Il est de plus impossible de trouver, parmi les données disponibles, une explication à ces variations, lorsqu'on compare les événements ou les bassins versants. Il est dangereux de vouloir extrapoler des résultats obtenus sur un site dans des conditions particulières à un autre site sous d'autres conditions.

Cela est particulièrement vrai pour les équations de régression établies site par site, polluant par polluant. Elles ont servi à reconstituer les caractéristiques des événements pluvieux qui manquaient (volume écoulé, concentration moyenne ou masse de pollution par événement). Les caractéristiques qui apparaissent dans les équations sont très variables d'un site à un autre. Il est impossible de relier plus particulièrement la concentration moyenne ou la masse rejetée pendant un événement pluvieux à telle ou telle variable. Le deuxième type de résultat qui présente une grande variabilité est la masse annuelle. Ces masses ont pu être évaluées pour chaque site grâce aux équations de régression. Leur emploi a permis d'établir des intervalles de confiance autour des valeurs. Ces intervalles sont souvent très larges. En effet, les ajustements obtenus ne sont pas tous de bonne qualité (peu de mesures disponibles ou événements non représentatifs). De plus, le nombre de sites est assez restreint. Par conséquent, la seule relation qui apparaît est celle qui est la plus évidente et qui existe entre les masses annuelles et les surfaces des sites.
Une grande variabilité se retrouve également lorsqu'on étudie la répartition de la masse en cours d'événement. Les formes sont très variables d'un événement à un autre, et sur un site donné. De plus, il ne semble pas y avoir de relations entre ces répartitions et les caractéristiques des événements pluvieux. En moyenne, la pollution contenue dans la première partie de l'écoulement est légèrement supérieure à celle de la deuxième partie. Il y a néanmoins un nombre non négligeable d'événements pour lesquels la majeure partie de la masse de pollution est rejetée en fin d'écoulement. Cela montre que le premier flot, très souvent considéré dans la littérature comme la fraction de l'écoulement la plus polluée, ne contient pas la plus grande fraction de la pollution rejetée par un événement. II ne peut pas être le seul facteur utilisé pour dimensionner les ouvrages de traitement.

Enfin, l'un des grands intérêts de la base est de fournir des indications sur les dimensions des ouvrages de traitement. Là encore les résultats sont très variables d'un site à un autre. Pour avoir un ordre d'idée, pour intercepter $80 \%$ de la masse annuelle il faut des volumes de stockage entre 50 et $150 \mathrm{~m}^{3} / \mathrm{h}$ a actif, et des débits de traitement entre 10 et $60 \mathrm{l} / \mathrm{s} / \mathrm{ha}$ actif. Les variations semblent moins provenir des caractéristiques des bassins versants que des périodes de retour des événements pluvieux qui ont été utilisés.

Enfin, les recommandations les plus importantes qui apparaissent à l'issue de ce travail sont les suivantes :

- les résultats obtenus à partir d'une campagne de mesure de quelques mois ne sont représentatifs que de cette campagne ;

- avec une dizaine d'événements, il est difficile d'extrapoler les résultats à d'autres sites ou d'autres événements ; - pour bien connaitre la pollution engendrée par un site et pour mettre en place un ouvrage de traitement réellement efficace, il est difficile de faire l'économie de mesures ;

- le phénomène de premier flot est trop rare pour être pris en compte lors du dimensionnement des ouvrages de traitement.

\section{RÉFÉRENCES BIBLIOGRAPHIQUES}

[1] Bertrand-Krajewski J.-L., Chebbo G., Saget A. (1995). Le premier flot (ou comment s'en débarrasser?). Document de travail pour le groupe de travail Pluvial AGHTM.

[2] Chebbo G., SAGet A. (1995), - Pollution of urban wet weather discharges. In : Encyclopedia of Environmental Biology. Academic Press lnc. Vol. 3, pp. 171-182.

13] Elubis J.B. (1985). - Pollutional Aspects of Urban Runoff In : Urban Runoff Pollution, H.C. Tomo, J. Marsalek and M. Desbordes (Eds). NATO ASI Series G: Vol 10 Springer-Verlag $1-38$.

14] GEIGER W.F. (1986). - Variation of combined runoff quality and resulting pollutant retention strategies. In : Proceedings of the international conference in Urban Storm Water Quality and Effects upon Receiving Waters, Wageningen, the Netherlands. F.H.M. van de Ven, I.C. Hooghart (Eds), The Hague, the Netherlands, pp. 71-91.

[5] Saget A., Chebbo G., Desbordes M. (1995). - Urban discharges during wet weather: What volumes have to be treated. In : Water Science and Technology, Vol. 32, No 1, pp. 225-232.

16] SAgET A. (1994). - Base de données sur la qualité des rejets urbains par temps de pluie: Distribution de la pollution rejetée, Dimensions des ouvrages d'interception. Thèse de Doctorat de l'Ecole Nationale des Ponts et Chaussées. 217 p. + annexes. 